\title{
Non-Gaussian Berkson Errors in Bioassay
}

\author{
Alaa Althubaiti \& Alexander Donev
}

First version: 12 May 2011

Research Report No. 2, 2011, Probability and Statistics Group

School of Mathematics, The University of Manchester 


\title{
Non-Gaussian Berkson Errors in Bioassay
}

\author{
Alaa Althubaiti and Alexander Donev* \\ School of Mathematics, University of Manchester, Oxford Road, Manchester M13 9PL, UK
}

\begin{abstract}
Summary
The experimental design plays an important role in every experimental study. However, if errors in the settings of the studied factors cannot be avoided, i.e. Berkson errors occur, the estimates of the model parameters may be biased and the variability in the study increased. Correction methods for the effect of Berkson errors are compared. The emphasis is on the study of correlated Berkson errors which follow non-Gaussian distribution as this appears to have been a neglected, yet important, area. We also examine independently distributed errors. It is shown that the regression calibration approach bias correction methods are useful when the Berkson errors are independent. However, when these errors are dependent, the newly proposed method B-SIMEX clearly outperforms the other methods.
\end{abstract}

Keywords: errors-in-variables, regression calibration, serial dilution designs, dilution errors, SIMEX, B-SIMEX.

*Corresponding author. Tel. +44 (0) 161306 3699. Fax: +44 (0) 1612755819.

E-mail addresses: A.N.Donev@manchester.ac.uk

Postal address: School of Mathematics, University of Manchester, Oxford Road, Manchester M13 9PL, UK. 


\section{Introduction}

Bioassays are experiments used for screening, testing and ranking of compounds in biological or medical research. Typically these studies are used to assess the potency and the side effects of the compounds which may be candidate or established drugs. The experimental design of such studies specifies the doses, or concentrations, for which the response of interest is measured. The data are then used to fit a statistical model, and the model parameters can be used to assess the effect of interest. The success of these studies depends on the experimental design and its implementation. This paper is concerned with the analysis of bioassay data when the required doses cannot be done accurately and the true doses are unknown and therefore cannot be used in the analysis of the data. This is a typical scenario of the so-called Berkson errors-in-variables (EIV) problem (Berkson, 1950). We are mainly concerned with the case when Berkson Gamma distributed EIV appear in bioassay preclinical studies, though the methodology that we propose can be easily extended to use in other bioassay studies, and indeed in many other cases when Berkson non-Gaussian (i.e. not normally distributed) EIV occur.

It is well known (see for example Carroll et al, 2006, for a comprehensive review of the research in this area) that the naive analysis ignoring the EIV leads to inaccurate assessment of the variability and often also to biased estimators of the model parameters. Most of the work that has been done is devoted to EIV in observational studies where classical EIV are assumed (Küchenhoff et al., 2007). Established bias correction techniques are the simulation-extrapolation (SIMEX), regression calibration, and the instrumental variable approach (Cook and Stefanski, 1994; Carroll and Stefanski, 1990; Greenland, 2000). These methods require some knowledge about the distribution of the EIV. In most cases the 
assumption of Gaussian EIV has been used. An exception is Wang et al. (2010), who considered the case of non-Gaussian EIV when a simple linear model has to be estimated.

The problem of EIV is less studied in designed experiments where the Berkson EIV are usually considered. Correction methods have been developed to produce analysis of the data that accounts for the effects of the EIV. Bias correction in the case of Berkson Gaussian EIV when linear or generalized linear models have to be estimated has been studied by Burr (1988), Rudemo et al. (1989), Buonaccorsi and Lin (2002), Kim et al. (2006), Küchenhoff et al. (2007) and Althubaiti and Donev (2010).

The effect of EIV in bioassay has received specific attention because of the importance of these studies (Racine-Poon et al. (1991), Dellaportas and Stephens (1995), Higgins et al. (1998), and Gelman et al. (2004)). Again, EIV with normal distribution has been assumed. It was reported that the naive analysis seriously underestimates the variability of the estimates of the parameters of the model and that the response variance is inflated. Racine-Poon et al. (1991) recognized that EIV are often proportional to the concentrations and developed a model to describe how the data are generated when a serial dilution design (SDD) is used and dilution errors in concentrations are made. In a SDD, the dilution process starts with preparing the top concentration $w_{1}$. The next dose is obtained by diluting the previous by a dilution factor $k$. The dilution process continues until the required number of doses is achieved. Wang and Davidian (1996) showed that only when the EIV are normally distributed with non-zero mean, the estimates of the model parameters are biased. However, no correction method was established to adjust for such a bias.

Non-Gaussian Berkson EIV have not been studied. However, the following example shows how such errors frequently occur in bioassays in early stages of the drug discovery process and therefore their study is important. 
Example 1. Dimethyl sulfoxide (DMSO) is a widely used solvent in bioassay because of its excellent solubilising ability, chemical inertness and high boiling and freezing points. However, it is also highly hydrophilic and will rapidly absorb water in many possible storing conditions. This may result in reduced stock concentration, compound precipitation, crystallization and degradation. As a result, when DMSO is used as a solvent in bioassay the doses of compounds could be lower compared to those specified by the experimental design and inaccurate data would be used in the naive statistical analysis ignoring the EIV.

Dawson and Donev (2007) present the results of a study on the stability of solutions for testing in bioassays when the compounds to be tested are dissolved in DMSO. The experiment aimed to quantify the dilution of compounds as a result of the absorption of water by DMSO. Compounds from different chemical series and molecular weight bands were used. Serial dilution designs were used in vivo to collect the data. These experimental designs are easy to use and have excellent statistical properties (Donev and Tobias, 2011).

The dilution by water uptake was measured by recording the volume gain during a typical laboratory storage period. Other possible reasons for reducing the intended doses that were mentioned earlier were not studied. Over the length of the study when the compounds were stored in room conditions, $11 \%$ - 16\% dilution was noted, while when they were stored in a fridge it was $10 \%$ - 24\%. Figure 1 shows the data when the solutions have been kept in a fridge. The actual difference between the target and the actual doses certainly depends on the exact time when the solutions have been used. The distribution of the resulting errors can be modelled in different ways, but a Gamma distribution seemed to suit well the collected data.

We study the effect of non-Gaussian errors in setting doses in bioassay and propose and compare correction methods aiming to reduce the effect of the EIV on the analysis. Two error models are investigated. The first error model assumes that the EIV are dependent. For example, such a model will be appropriate when a SDD is used and errors occur in the top 
doses, which then propagate to all other doses. This would be the typical scenario wherein the DMSO is used to obtain the initial concentration. The second error model assumes that all doses in the experimental design are subject to independent errors. Such a scenario can occur, for example, in animal studies where each dose is prepared prior to administration to each animal. Motivated by Example 1 we focus on the case when the EIV are negative with Gamma distribution. However, the proposed methodology can be extended to other distributions and error models.

Section 2 describes the problem of errors in doses in bioassays and uses Monte Carlo simulations to study their impact on the results in a statistical analysis that ignores them. It also provides details about the studied error models. Section 3 presents two approaches for correcting the effect of EIV on the analysis of data. One of them is based on regression calibration. The second method extends the idea of the SIMEX method for the case when the EIV are of Berkson's type. This new method, which we call B-SIMEX, is particularly effective when the EIV are correlated. The paper concludes with a comparison of the two approaches.

\section{Effect of Berkson errors on statistical analysis of bioassay results}

\subsection{Modelling bioassay data with EIV}

In a typical bioassay, a response of interest, say $y$, is measured at different doses, say $w_{1}, \ldots, w_{n}$, of a studied compound. The equation proposed by Hill (1913)

$$
y_{t}=f\left(w_{t}, \boldsymbol{\beta}\right)=\beta_{3}+\frac{\beta_{4}-\beta_{3}}{1+\left(\frac{w_{t}}{10^{\beta_{1}}}\right)^{\beta_{2}}}+\varepsilon_{t} \text {, }
$$


$t=1,2, \mathrm{~K}, n$, is usually used in the analysis of the data. In (1) $y_{t}$ is the response corresponding to dose $w_{t}, \boldsymbol{\beta}=\left(\beta_{1}, \beta_{2}, \beta_{3}, \beta_{4}\right)^{\mathrm{T}}$ is a vector of the model parameters where $\beta_{1}$ is the logarithm to base 10 of the dose required to achieve a response half way between the maximum response $\beta_{4}$ and the minimum response $\beta_{3}$, and $\beta_{2}$ is called the Hill slope. In order to obtain good estimates for $\beta_{3}$ and $\beta_{4}$, positive and negative control measurements are also collected. We focus on the case when the errors $\varepsilon_{t}, t=1,2, \mathrm{~K}, n$, can be assumed to be additive and independent and to follow a normal distribution with zero mean and constant variance $\sigma_{\varepsilon}^{2}$. Situations when the assumptions about the error structure are different can be handled in a way similar to that proposed in this paper.

Primarily, the interest is in estimating $\beta_{1}$, commonly referred to as $\log \mathrm{IC}_{50}$ or $\log \mathrm{EC}_{50}$, as it provides guidance about the potency of a studied compound. Small values of $\beta_{1}$ indicate high potency of the studied compound. The accuracy of the estimate of $\sigma_{\varepsilon}^{2}$ is also very important as it is used in the statistical inference required for the study.

As discussed earlier, EIV in bioassay may arise due to different reasons. Without loss of generality, we focus on two typical situations. For each of them we define a model for the effects of the EIV. Example 1 provides motivation for these models.

Error model 1. In this case we assume that the top dose $w_{1}$ of a SDD is subject to error. This error propagates to the other doses in the experimental design but no significant additional errors in the doses are made.

If the error at the top dose is $u_{1}$, with mean $m_{u_{1}}$ and variance $\sigma_{u_{1}}^{2}$ the actual concentrations are given by

$$
x_{t}=w_{t}-k^{-(t-1)} w_{1} u_{1},
$$

$t=1,2, \mathrm{~K}, n$. Hence, 


$$
\begin{aligned}
E\left[x_{t} \mid w_{t}\right] & =w_{t}-k^{-(t-1)} w_{1} m_{u_{1}}=w_{t}-w_{t} m_{u_{1}}, \\
\operatorname{var}\left(x_{t} \mid w_{t}\right) & =k^{-2(t-1)} w_{1}^{2} \sigma_{u_{1}}^{2},
\end{aligned}
$$

$t=1,2, \mathrm{~K}, n$. Also, $\operatorname{cov}\left(u_{t}, \varepsilon_{t}\right)=0$, while for doses $x_{t}$ and $x_{v}, t, v=1,2, \mathrm{~K}, n, t \neq v$,

$$
\operatorname{cov}\left(x_{t}, x_{v}\right)=k^{-[(t-1)+(v-1)]} w_{1}^{2} \sigma_{u_{1}}^{2}=k^{-(t+v-2)} w_{1}^{2} \sigma_{u_{1}}^{2} .
$$

Therefore the actual doses in the design are heteroscedastic and dependent random variables.

Error model 2. In this case we assume that each dose is obtained independently from the other doses and therefore the EIV occur independently from each other. Then the actual dose $x_{t}$ is given by

$$
x_{t}=w_{t}-w_{t} u_{t}
$$

$t=1,2, \mathrm{~K}, n$, where the errors $u_{t}$, are independent and identically distributed random variables with mean $m_{u}$ and a common variance $\sigma_{u}^{2}$. Therefore

$$
\begin{aligned}
E\left[x_{t} \mid w_{t}\right] & =w_{t}-w_{t} m_{u} \\
\operatorname{var}\left(x_{t} \mid w_{t}\right) & =w_{t}^{2} \sigma_{u}^{2},
\end{aligned}
$$

$t=1,2, \mathrm{~K}, n$. Also, $\operatorname{cov}\left(u_{t}, \varepsilon_{t}\right)=0$, and for doses $x_{t}$ and $x_{v}, t, v=1,2, \mathrm{~K}, n, t \neq v$,

$$
\operatorname{cov}\left(x_{t}, x_{v}\right)=0 .
$$

Thus, if independent errors occur in concentrations of a SDD, the actual concentrations of the design are heteroscedastic and independent random variables. Note both the expectation and variance of the actual doses in case of independent and dependent errors are equivalent only if $\sigma_{u_{1}}^{2}=\sigma_{u}^{2}$. 


\subsection{Effect of EIV on the estimates of $\beta_{1}$}

We use Monte Carlo simulations to evaluate the effect of EIV on the estimates of $\beta_{1}$. In the following examples we assume that the EIV following the error models described in the previous section are negative with Gamma distribution $u_{1} \sim \Gamma(\gamma, \delta)$. The parameters of their distribution are set to $\gamma=1$ and $\delta=\sigma_{u_{1}}$, i.e. $u_{1} \sim \Gamma\left(1, \sigma_{u_{1}}\right)$.

Example 2. We assume that the EIV follow Error model 1 (i.e. D-G EIV). SDD with 8 doses, $w_{1}=32, k=2$, positive and negative controls, and the Hill equation (1) with values $\beta_{1}=$ $0.301, \beta_{2}=-1.0, \beta_{3}=363$, and $\beta_{4}=700$ generated the data. These values were chosen to be similar to those estimated for a compound in the experiment reported by Dawson and Donev (2007). However, the experimental error was kept small in order to prevent it from obscuring the effect of EIV, i.e. $\varepsilon \sim N(0,0.01)$. These results were also later confirmed by computer simulation when the values of $\sigma_{\varepsilon}^{2}$ were larger, but these results are omitted here.

Simple algebra can be used to show that $\gamma$ and $\delta$ of all doses in the experimental design are $\left[w_{t}-w_{t} \sigma_{u_{1}}\right]^{2}\left[w_{t}^{2} \sigma_{u_{1}}^{2}\right]^{-1}$ and $w_{t}^{2} \sigma_{u_{1}}^{2}\left[w_{t}-w_{t} \sigma_{u_{1}}\right]^{-1}, t=1,2, \mathrm{~K}, n$, respectively. EIV with $\sigma_{u_{1}}=0.023$ and $\sigma_{u_{1}}=0.044$, were generated.

The results were based on $10^{4}$ simulations. Naive and actual analyses of the simulated data were carried out. Table 1 shows the following summaries of the results:

- the percentage of the mean and median relative bias for $\hat{\beta}_{1}$, given by $\left|E\left[\hat{\beta}_{1}-\beta_{1}\right] / \beta_{1}\right| \times 100$ and $\left|\operatorname{median}\left[\hat{\beta}_{1}-\beta_{1}\right] / \beta_{1}\right| \times 100$, respectively,

- the averaged model based standard error (SE), based on the linearized Fisher's information matrix,

- the empirical Monte Carlo model standard error, 
- the relative model standard errors (the ratio of the averaged model based standard error to the Monte Carlo standard error).

Certainly, when the median and the mean biases are large, the interpretation of the results could be misleading. This also extends to the cases when $\sigma_{u_{1}}$ is underestimated or overestimated. Then the relative standard error is smaller or larger than 1.

The results show that $\hat{\beta}_{1 \text {,naive }}$ is asymptotically biased. For example, when $\sigma_{u_{1}}=0.023$, the absolute median and mean relative biases are $2.344 \%$ and $3.404 \%$, respectively. The bias increases with the error variance: when $\sigma_{u_{1}}=0.044$, the absolute median and mean relative biases are $4.516 \%$ and $6.697 \%$ respectively. The naive estimate underestimates the true potency.

The EIV lead to increased variability, as the standard error is increased from $4.550 \times 10^{-4}$ to $1.475 \times 10^{-2}$. However, the naive analysis fails to identify that and considerably underestimates it. Therefore the relative model standard error is just $3.086 \times 10^{-2}$. In short, in the case of D-G errors, $\hat{\beta}_{1, \text { naive }}$ are asymptotically biased and inefficient, while $\hat{\sigma}_{\varepsilon}^{2}$ underestimates $\sigma_{\varepsilon}^{2}$.

Example 3. We consider again the experimental situation described in Example 2. However, this time the errors in the concentrations are independent gamma distributed and follow Error model 2 (I-G EIV). Hence, in this case the actual concentrations are smaller than the target concentrations and are generated by (3). Consequently, the actual concentrations follow a gamma distribution with parameters $\left[w_{t}-w_{t} \sigma_{u}\right]^{2}\left[w_{t}^{2} \sigma_{u}^{2}\right]^{-1}$ and $w_{t}^{2} \sigma_{u}^{2}\left[w_{t}-w_{t} \sigma_{u}\right]^{-1}$, $t=1,2, \mathrm{~K}, n$, respectively. 
Table 2 summarizes the results based on $10^{4}$ simulations. The bias of $\hat{\beta}_{1, \text { naive }}$ is similar but slightly higher than for D-G EIV. The increase in variability due to EIV is also comparable to that noted in Example 2. However, I-G EIV seem to lead to more efficient and consistent naive estimates than when there are D-G EIV.

\section{Adjusting for Non-Gaussian Berkson Errors}

We start this section by briefly describing two commonly used methods for correcting the effect of EIV, the regression calibration and the simulation-extrapolation method, and discuss their suitability to deal with the Berkson EIV. Then we propose an approach similar to SIMEX that is specifically designed to deal with non-Gaussian Berkson errors. We compare it with the Regression Calibration when both approaches are applied to the situations discussed in Examples 2 and 3.

\subsection{Regression Calibration and SIMEX}

Regression calibration is a simple but effective way of adjusting for the effect of EIV. It has been extensively used in the literature on EIV models, for example, in epidemiology studies with Cox proportional hazards models (Prentice, 1982), in generalized linear models (Armstrong, 1985; Rosner et al., 1989; Schafer, 1990; Kuha, 1994), and in nonlinear response models (Gleser, 1990). The basic idea of RC is to replace the observed predictor in the regression model with an unbiased estimate of its true value. The estimate can be found in several ways, but the most common method is to approximate the conditional expectation of the true predictor given the observed value $E[x \mid w, z]$, where $z$ is any other covariate in the model (Carroll et al., 2006). The expectation can be found either analytically (precisely or approximately using the delta method (Meyer, 1965 p.128)), or empirically by computer simulations. 
Estimating the expectation $E[x \mid w, z]$ has been described as an "art” (Carroll et al. 2006) as it requires a full understanding of the EIV model. The regression calibration approach can be used when Berkson EIV occur if a direct estimation of $E[x \mid w, z]$ can be obtained. The regression calibration method can also be used with non-Gaussian EIV (see for example Suh and Schafer, 2002). However, although RC is widely applicable, the consistency of the approach is questionable when dealing with complex nonlinear models, and in most cases a small variance of EIV is required for the approximation to be satisfactory. In addition, heteroscedastic EIV could affect the robustness of the approach. For example, if the variance of the true value, given the observed one, $\operatorname{var}(x \mid w)$, is heteroscedastic, RC may only be useful for correcting the bias in naive estimates; however, it does not provide efficient standard errors of the parameter estimates (Carroll et al., 2006). This may occur when dealing with Berkson errors since they are often heteroscedastic by nature, that is, the error in the input of a design is mostly correlated to the design value. For example, Althubaiti and Donev (2010) show that the efficiency of the regression calibration is improved if the weighted least squares estimation of the model parameters is used in combination with the regression calibration. This extends the result of Carroll et al. (page 80, 2006) for the case of Berkson EIV. The inverse of the variances of the responses at each design point are used as weights. Comparisons between the results with reference to analytical and empirical estimates of the calibrated design matrix were also obtained. The estimates that were obtained this way were found to be approximately unbiased and efficient. However, Althubaiti and Donev (2010) limit their study to independent, normally distributed EIV.

The simulation-extrapolation approach was proposed by Cook and Stefanski (1994) as a functional correction tool for classical EIV effects on linear and nonlinear models. It produces the so-called SIMEX estimator which is then used to correct the bias in the naive parameter estimates. The algorithm is based on estimating the regression coefficients through a process 
of adding errors with increasing variances to the observable variables that have been measured with errors. The relationships between the error variances and the estimated parameters are modelled separately and then extrapolated to the ideal case where there are no EIV. The algorithm works as follows.

Assume the th observation is measured with a classical error

$$
w_{t}=x_{t}+u_{t}
$$

where $x_{t}$ is the actual unknown th measurement, $u_{t}$ is an independent distributed random variable with mean zero and variance $\sigma_{u}^{2}$. Suppose the parameters of the model are given by the vector $\boldsymbol{\beta}$. In the simulation step of SIMEX, $n$ new observations of observed predictor $w$ are generated for $B$ times by

$$
w_{b, t}=w_{t}+\lambda^{1 / 2} u_{b, t}, \quad t=1,2, \mathrm{~K}, n, \quad b=1,2, \mathrm{~K}, B,
$$

where $\lambda \geq 0$ is called the multiplication factor, and it defines the amount of additional errors added to the observed variable, $u_{b, t}$ is independent and identically distributed, normal random variables with mean zero and variance $\sigma_{u}^{2}$, i.e. $u_{b, t} \sim N\left(0, \sigma_{u}^{2}\right)$, and $B$ is the number of samples being generated in the simulation process. The new values of the predictor are analysed by finding the regression parameter estimates $\hat{\boldsymbol{\beta}}_{b}(\lambda)$ of regressing $y$ on $w_{t, b}$ for each value of $\lambda$, where $\hat{\boldsymbol{\beta}}_{b}(\lambda)$ is a vector of the naive ordinary least squares estimates of the model parameters. After obtaining the estimates $\hat{\boldsymbol{\beta}}_{b}(\lambda)$, the average of these estimates is computed for each $\lambda$ by $\hat{\boldsymbol{\beta}}(\lambda)=\frac{1}{B} \sum_{b=1}^{B} \hat{\boldsymbol{\beta}}_{b}(\lambda)$. A model can be then fitted for the components of $\hat{\boldsymbol{\beta}}(\lambda), \lambda \geq 0$ as a function of $\lambda$. From (4), it follows that the total measurement error variance for the th set of observations is 


$$
\operatorname{var}\left[w_{b, t}(\lambda) \mid x_{t}\right]=(1+\lambda) \sigma_{u}^{2}
$$

Hence, when $\lambda=0$, the model is free from additional errors but still contains the original errors (naive model) and the estimate found at that point is the naive estimator $\hat{\boldsymbol{\beta}}_{\text {naive }}$. The extrapolation in SIMEX is established by setting $\lambda=-1$ in the fitted model for each component of the vector $\hat{\boldsymbol{\beta}}(\lambda)$ as then, from (5), the total measurement error variance in $w_{b, t}$ is zero. Cook and Stefanski (1994) used three types of fitting extrapolation functions, the simple linear, quadratic model, and nonlinear model. The exact extrapolation function is usually unknown. SIMEX is only approximately adjusting the bias in the naive estimators. The method has usually been applied with the assumption of normally distributed measurement errors but this assumption is not essential (Carroll et al., 1996).

However, SIMEX is not a suitable method to correct Berkson EIV following the error models discussed earlier. When the errors are asymmetric, the consistency of SIMEX's estimator is reduced (Cook \& Stefanski, 1994) and (Wang et al., 2010). Also, SIMEX has been mainly used when the assumption of classical EIV holds since it may produce untrue results when dealing with Berkson errors wherein the errors are normally distributed. In case of classical errors, the observed variables contain the EIV and the estimates of their coefficients are biased. Thus, adding additional errors to these variables with increasing variances leads to increasingly biased estimates, in the same direction of the bias in the naive estimates. However, this is not necessary true in Berkson error models since the actual analysis is the one containing the errors and the observed variable is error free. Hence, adding Gaussian errors with increasing variances to the design points, does not guarantee that increasingly or decreasingly biased estimates will be obtained, making the main idea of SIMEX inapplicable. 
In the following section we propose an alternative method for simulation-extrapolation that can be used to correct for non-Gaussian EIV. It is particularly effective when these errors are correlated, e.g. follow Error model 1. The new approach is straightforward and easy to implement.

\subsection{Simulation-Extrapolation Method for Adjusting for Berkson Non-Gaussian EIV (B- SIMEX)}

We illustrate the B-SIMEX method using the assumption of D-G errors. However, the approach can be applicable whenever the assumption of Berkson EIV, follow asymmetric distribution holds. Similar to SIMEX, B-SIMEX requires the distribution of the EIV to be known. In the simulations stage randomly generated errors with sign opposite to that of the actual EIV are added to the values specified by the experimental design.

For instance, in Example 2 where the errors in concentrations are negative and D-G, since the actual doses are generated by model (2), in the simulation step, $B$ new values $x_{b, t}^{*}(\lambda)$ are generated for several values of $\lambda, \lambda \geq 0$, by using $x_{b, t}^{*}(\lambda)=w_{t}+\lambda^{1 / 2} w_{t} u_{b, 1}, t=1,2, \mathrm{~K}, n$, $b=1,2, \mathrm{~K}, B$. Note that the increasing amounts of errors are added to the target (error free) observations, where $u_{b, 1}, b=1,2, \mathrm{~K}, B$ are identically independent random errors, simulated from an asymmetric distribution with variance $\sigma_{u_{1}}^{2}$, the generated values $x_{b, t}^{*}(\lambda)$, $t=1,2, \mathrm{~K}, n, b=1,2, \mathrm{~K}, B$, are constantly larger than the target concentrations, and have the same distribution of the errors $u_{b, 1}, b=1,2, \mathrm{~K}, B$, with variance

$$
\operatorname{var}\left[x_{b, t}^{*}(\lambda) \mid w_{t}\right]=\lambda k^{-2(t-1)} w_{1}^{2} \sigma_{u_{1}}^{2}=\lambda \operatorname{var}\left(x_{t} \mid w_{t}\right), \quad t=1,2, \mathrm{~K}, n .
$$

Thus, the variance of the generated doses $x_{b, t}^{*}(\lambda)$ is equal to the variance of the actual concentration multiplied by the factor $\lambda$. Similar to the extrapolation step in SIMEX, the 
parameter vector $\hat{\boldsymbol{\beta}}(\lambda)$ is modelled as a function in $\lambda$. The relation is then extrapolated by setting $\lambda=-1$ to obtain a B-SIMEX estimator $\hat{\boldsymbol{\beta}}_{b-\text { simex }}$.

As with SIMEX, it is important to point out that the consistency of B-SIMEX is dependent on the appropriate choice of the function that describes the relation between $\hat{\boldsymbol{\beta}}(\lambda)$ and $\lambda$. Linear, quadratic and nonlinear extrapolation functions used by SIMEX can be used by B-SIMEX too. The variance of the B-SIMEX estimates can be calculated using the method of simulation-extrapolation variance estimation (Stefanski and Cook, 1995). The approach produces approximately unbiased and efficient estimates.

Example 4. A Monte Carlo simulation was conducted to examine a typical situation in practice wherein B-SIMEX estimation is applied. The aim of the simulation is to examine the adjustment of B-SIMEX on the bias of the naive estimators, as a result of negative D-G EIV. In the simulation, we use the same assumptions for the model parameters, and simulation setting in Example 2, but only one simulation is carried out. The multiplicative factor $\lambda$ was set to $0,0.5,1,1.5$, and 2 , and $B=1500$ samples were generated in order to obtain the extrapolation function. We choose the number of simulated samples in the simulation step of B-SIMEX to be relatively large, in order to minimize the amount of variability in the simulation. Smaller values of $B$ (e.g. 500) were usually sufficient. For the used simulation settings, the calculations take around one minute, so choosing smaller value of $B$ would usually be unnecessary.

Figure 2 shows the results of the calculations. Clearly, the B-SIMEX estimate is considerably closer to the actual than the naive one. In the next section we study the asymptotic properties of the B-SIMEX estimates and compare them with those obtained using regression calibration. 


\subsection{Comparison of Calibration Approaches and B-SIMEX}

We extend Example 2 and Example 3, and use computer simulations to compare the actual and the naive estimates of $\beta_{1}$ with estimates obtained using B-SIMEX, regression calibration estimating $E\left[x_{t} \mid w_{t}\right]$ analytically $\left(\mathrm{RC}_{\mathrm{E}}\right)$ and empirically $\left(\mathrm{RC}_{\mathrm{A}}\right)$ and weighted regression calibration $\left(W R C_{E}\right.$ and $W R C_{A}$, respectively). An empirical estimate of the calibrated design matrix was computed over $10^{4}$ simulations. This number was seen large enough to achieve convergence. For the WRC approaches, the weights were also computed over $10^{4}$ simulations. The response error variance is assumed to be known or well estimated prior to the analysis and its value was $\sigma_{\varepsilon}^{2}=0.01$.

Example 2 (continued). The results of simulations in the presence of D-G EIV are given in Table 3. For the purpose of comparisons linear, nonlinear and quadratic extrapolation functions were applied. The linear extrapolation was found to give more consistent estimators than the other extrapolation functions. Therefore we only report the simulation results when it is used. The results show that B-SIMEX reduces considerably the bias of the estimate of $\beta_{1}$. For example, when $\sigma_{u_{1}}=0.023$, the percentage of absolute relative median bias of $\hat{\beta}_{1, b-\text { simex }}$ was 0.856 , while the percentage of absolute relative median bias of $\hat{\beta}_{1 \text {,naive }}$ was 2.344 . The biases reduction obtained using regression calibration, while smaller than that achieved by BSIMEX, were also noteworthy. Interestingly, the corresponding biases when the estimates were obtained by empirical regression calibration $\mathrm{RC}_{\mathrm{E}}$ and $\mathrm{WRC}_{\mathrm{E}}, 0.974$ and 0.986 , respectively, were smaller than those obtained when analytical regression calibration $\left(\mathrm{RC}_{\mathrm{A}}\right.$ and $\mathrm{WRC}_{\mathrm{A}}$ ) was used, 1.013 and 1.025, respectively. The simulation results show that the BSIMEX estimates follow asymptotically the distribution on the EIV, the Gamma distribution.

The average model based standard errors obtained in the naive and the actual analyses were identical $\left(4.550 \times 10^{-4}\right)$, while the Monte Carlo standard error was considerably higher 
$\left(1.475 \times 10^{-2}\right)$. This shows that ignoring the EIV would result in using wrong estimates of $\sigma_{\varepsilon}$ in the statistical inference of the results. This problem is also associated with the estimates of $\sigma_{\varepsilon}$ obtained using a regression calibration approach as in all studied cases the relative standard errors were very small. However, the estimate of $\sigma_{\varepsilon}$ obtained using B-SIMEX was fairly accurate and the relative standard error was just 0.601 .

The results when $\sigma_{u_{1}}=0.044$ confirm the conclusions when $\sigma_{u_{1}}=0.023$ and show that larger EIV have larger impact on the results obtained in the statistical analysis in the presence of EIV.

Example 3 (continued). We compare the efficiency of B-SIMEX and regression calibration to correct the effect of I-G EIV in the same way as above. The results of the simulations are given in Table 4. B-SIMEX again reduced the bias of the estimate of $\beta_{1}$. However, regression calibration adjustments were considerably better. The empirical weighted regression calibration reduced the bias to just 0.132 when $\sigma_{u}=0.023$, and to 0.189 when $\sigma_{u}=0.044$. The Monte Carlo standard error estimate is $2.360 \times 10^{-2}$ in the naive statistical analysis showed an increase in the variability similar to that when D-G EIV occurred in Example 2. As expected, the reduction in the bias of $\beta_{1}$ reduced these values. However, the results in Table 4 show that even when the bias of $\beta_{1}$ was reduced, i.e. when $\mathrm{WRC}_{\mathrm{E}}$ and $\mathrm{WRC}_{\mathrm{A}}$ were used, the averaged model-based standard error and the Monte Carlo standard error remained relatively large. The relative standard error when regression calibration methods were used were closer to 1 than when B-SIMEX was used, showing that in the case of independent EIV regression calibration led to better results.

The variability of the bias correction with B-SIMEX is somewhat smaller than that of the naive estimator. This result shows how B-SIMEX and SIMEX differ as the later approach 
is commonly known to produce an estimator with larger variability than that of the naive one, a phenomena that has been established in classical EIV models and called bias-variance tradeoff.

The robustness of the B-SIMEX estimates was studied too. In separate simulations smaller and larger (by the factor 2) variances of G-D EIV than the true ones were used to obtain B-SIMEX estimates of $\beta_{1}$. It was noted that the B-SIMEX correction was more affected by underestimating than overestimating the variance of the EIV.

\section{Discussion}

The results presented in Section 2 show that Gamma distributed EIV lead to bias in the estimates of the model parameters and increased variability. Limited studies of other asymmetric distributions for the EIV confirm that this is the effect of such EIV in general. The comparison of the bias correction methods given in Section 3 shows that the asymptotic bias and the study variability can be considerably reduced when the error model is well understood and the distribution of the EIV is known. Methods based on the regression calibration approach appear to be particularly effective when the EIV are independent. In this case, the asymptotic bias in the estimates of the model parameters is almost eliminated. The empirical regression calibration turns out to be slightly but consistently better than that based on analytical approximation of the expected values of the model regressors. However, the increase in the variability caused by the EIV remains unaffected by the bias correction.

The regression calibration is also useful tool for bias correction when the EIV are dependent. However, $\sigma_{\varepsilon}$ is underestimated and therefore the interpretation of the results obtained using such methods could be misleading. The new approach presented in this paper, B-SIMEX, performs much better in such cases as it greatly reduces the asymptotic bias of the estimates of the model parameters and the asymptotic estimate of $\sigma_{\varepsilon}$ is unbiased. In short, 
we recommend B-SIMEX when the non-Gaussian EIV are dependent, but empirical regression calibration when they are independent.

\section{References}

1. Armstrong, B. (1985). Measurement error in generalized linear models. Communications in Statistics, Series B, 14, 529-544.

2. Berkson, J. (1950). Are there two regressions? Journal of the American Statistical Association, 45, 164-180.

3. Buonaccorsi, J.P., \& Lin C.-D. (2002). Berkson measurement error in designed repeated measures studies with random coefficients. Journal of Statistical Planning and Inference, 104, 53-72.

4. Burr, D. (1988). On errors-in-variables in binary regression-Berkson case. Journal of the American Statistical Association, 83, 739- 743.

5. Carroll, R. J., Ruppert, D., Stefanski, L. A., \& Crainiceanu, C. M. (2006). Measurement Error in Nonlinear Models: A Modern Perspective (2nd ed.). New York: Chapman and Hall/CRC.

6. Carroll, R.J., \& Stefanski, L.A. (1990). Approximate quasi-likelihood estimation in models with surrogate predictors. Journal of the American Statistical Association, 85, $652-663$.

7. Cook, J.R., \& Stefanski, L.A. (1994). Simulation-Extrapolation estimation in parametric measurement error models. Journal of American Statistical Association, 89, 1314-1328.

8. Dawson, J., \& Donev, A. (2007). Local compound storage: practical and statistical implications. Retrieved February 5, 2010, from http://www.spodtechnology.com/astrezeneca.php

9. Dellaportas, P., \& Stephens, D.A. (1995). Bayesian analysis of errors-in-variables regression models. Biometrics, 51, 1085-1095.

10. Donev, A. N. and Tobias, R. (2011). Optimal Serial Dilutions Designs For Drug Discovery Experiments, Journal of Biopharmaceutical Statistics, 21, 484-497.

11. Gelman, A., Chew, G. L., \& Shnaidman, M. (2004). Bayesian analysis of serial dilution assays. Biometrics, 60, 407-417.

12. Greenland, S. (2000). An introduction of instrumental variables for epidemiologists. Internaltional Journal of Epidemiology, 29, 722-729. 
13. Higgins, K.M., Davidian, M., Chew, G., \& Burge, H. (1998). The effect of serial dilution error on calibration inference in immunoassay. Biometrics, 54, 19-32.

14. Hill, A.V. (1913). The combinations of hemoglobin with oxygen and with carbon monoxide. Biochemical Journal, 7, 471-480.

15. Kim, H.M., Yasui, Y., \& Burstyn, I. (2006). Attenuation in risk estimates in logistic and cox proportional-hazards models due to group-based exposure assessment strategy. The Annals of Occupational Hygiene, 50, 623-635.

16. Kuha, J. (1994). Corrections for exposure measurement error in logistic regression models with an application to nutritional data. Statistics in Medicine, 13, 1135-1148.

17. Küchenhoff, H., Mwalili, S. M., \& Lesaffre, E. (2006). A general method for dealing with misclassification in regression: The misclassification SIMEX. Biometrics, 62, 8596.

18. Meyer, P.L. (1965). Introductory probability and statistical applications. London: Addison-Wesley.

19. Racine-Poon, A., Weihs, C., \& Smith, A. F. M. (1991). Estimation of relative potency with sequential dilution errors in radioimmunoassay. Biometrics, 47, 1235-1246.

20. Rosner, B., Willett, W.C., \& Spiegelman, D. (1989). Correction of logistic relative risk estimates for non-random measurement error. Statistics in Medicine, 8, 10511069.

21. Prentice, R. L. (1982). Covariate measurement errors and parameter estimation in failure time regression models. Biometrika, 69, 331-342.

22. Rudemo, M. Ruppert, D., \& Streibig, J.C. (1989). Random-effect models in nonlinear regression with application to bioassay. Biometrics, 45, 349-362.

23. Schafer, D.W. (1990). Measurement Error Model Estimation Using Iteratively Weighted Least Squares. In P. J. Brown \& W. A. Fuller (Eds.), Statistical Analysis of Measurement Error Models and Applications. American Mathematics Society: Providence.

24. Suh, E.-Y., \& Schafer, D.W. (2002). Semiparametric maximum likelihood for nonlinear regression with measurement errors. Biometrics, 58, 448-453.

25. Wang, N., \& Davidian, M. (1996). A note on covariate measurement error in nonlinear mixed effects models. Biometrika, 83, 801-812.

26. Wang, X.-F., Fan, Z., \& Wang, B. (2010). Estimating smooth distribution function in the presence of heteroscedastic measurement errors. Computational Statistics and Data Analysis, 54, 25-36. 
Table 1. D-G EIV: summary measures for the naive and actual estimators.

\begin{tabular}{cccc}
\hline Error size & Measures & Actual & Naive \\
\hline & $\mid$ Median relative bias for $\hat{\beta}_{1} \mid \times 100$ & $2.877 \times 10^{-2}$ & 2.344 \\
& $\mid$ Mean relative bias for $\hat{\beta}_{1} \mid \times 100$ & $2.708 \times 10^{-2}$ & 3.404 \\
$\sigma_{u}=0.023$ & Averaged model-based SE & $4.550 \times 10^{-4}$ & $4.550 \times 10^{-4}$ \\
& MC SE & $4.814 \times 10^{-4}$ & $1.475 \times 10^{-2}$ \\
& Relative SE & 0.945 & $3.086 \times 10^{-2}$ \\
\hline Error size & Measures & Actual & Naive \\
\hline & $\mid$ Median relative bias for $\hat{\beta}_{1} \mid \times 100$ & $2.663 \times 10^{-2}$ & 4.516 \\
$\sigma_{u}=0.044$ & $\mid$ Mean relative bias for $\hat{\beta}_{1} \mid \times 100$ & $2.534 \times 10^{-2}$ & 6.697 \\
& Averaged model-based SE & $4.551 \times 10^{-4}$ & $4.551 \times 10^{-4}$ \\
& MC SE & $4.808 \times 10^{-4}$ & $2.933 \times 10^{-2}$ \\
& Relative SE & 0.947 & $1.552 \times 10^{-2}$ \\
\hline
\end{tabular}

Table 2 . I-G EIV: summary measures for the naive and actual estimators.

\begin{tabular}{cccc|}
\hline Error size & Measures & Actual & Naive \\
\hline & $\mid$ Median relative bias for $\hat{\beta}_{1} \mid \times 100$ & $2.313 \times 10^{-2}$ & 2.978 \\
& $\mid$ Mean relative bias for $\hat{\beta}_{1} \mid \times 100$ & $2.259 \times 10^{-2}$ & 3.375 \\
$\sigma_{u}=0.023$ & Averaged model-based SE & $4.588 \times 10^{-4}$ & $5.533 \times 10^{-3}$ \\
& MC SE & $4.793 \times 10^{-4}$ & $1.192 \times 10^{-2}$ \\
& Relative SE & 0.957 & 0.464 \\
\cline { 2 - 4 } & Measures & Actual & Naive \\
\hline \multirow{5}{*}{$\sigma_{u}=0.044$} & $\mid$ Median relative bias for $\hat{\beta}_{1} \mid \times 100$ & $2.085 \times 10^{-2}$ & 5.781 \\
& $\mid$ Mean relative bias for $\hat{\beta}_{1} \mid \times 100$ & $2.120 \times 10^{-2}$ & 6.603 \\
& Averaged model-based SE & $4.610 \times 10^{-4}$ & $1.103 \times 10^{-2}$ \\
& MC SE & $4.806 \times 10^{-4}$ & $2.360 \times 10^{-2}$ \\
& Relative SE & 0.959 & 0.467 \\
\hline
\end{tabular}


Table 3. D-G EIV: summary measures for the naive, actual, B-SIMEX, RC, and WRC estimators.

\begin{tabular}{|c|c|c|c|c|c|c|c|c|}
\hline Error size & Measures & Actual & Naive & BSIMEX & $\mathrm{RC}_{\mathrm{E}}$ & $\mathrm{RC}_{\mathrm{A}}$ & $\mathrm{WRC}_{\mathrm{E}}$ & $\mathrm{WRC}_{\mathrm{A}}$ \\
\hline \multirow{5}{*}{$\sigma_{u}=0.023$} & $\mid$ Median relative bias for $\hat{\beta}_{1} \mid \times 100$ & $2.877 \times 10^{-2}$ & 2.344 & 0.856 & 0.974 & 1.013 & 0.986 & 1.025 \\
\hline & $\mid$ Mean relative bias for $\hat{\beta}_{1} \mid \times 100$ & $2.708 \times 10^{-2}$ & 3.404 & 1.919 & $8.612 \times 10^{-2}$ & $4.741 \times 10^{-2}$ & $7.976 \times 10^{-2}$ & $4.106 \times 10^{-2}$ \\
\hline & Averaged model-based SE & $4.550 \times 10^{-4}$ & $4.550 \times 10^{-4}$ & $2.008 \times 10^{-2}$ & $4.550 \times 10^{-4}$ & $4.550 \times 10^{-4}$ & $3.934 \times 10^{-4}$ & $3.934 \times 10^{-4}$ \\
\hline & MC SE & $4.814 \times 10^{-4}$ & $1.475 \times 10^{-2}$ & $1.208 \times 10^{-2}$ & $1.060 \times 10^{-2}$ & $1.060 \times 10^{-2}$ & $1.061 \times 10^{-2}$ & $1.061 \times 10^{-2}$ \\
\hline & Relative SE & 0.945 & $3.086 \times 10^{-2}$ & 0.601 & $4.291 \times 10^{-2}$ & $4.292 \times 10^{-2}$ & $3.707 \times 10^{-2}$ & $3.708 \times 10^{-2}$ \\
\hline Error size & Measures & Actual & Naive & BSIMEX & $\mathrm{RC}_{\mathrm{E}}$ & $\mathrm{RC}_{\mathrm{A}}$ & $\mathrm{WRC}_{\mathrm{E}}$ & $\mathrm{WRC}_{\mathrm{A}}$ \\
\hline \multirow{5}{*}{$\sigma_{u}=0.044$} & $\mid$ Median relative bias for $\hat{\beta}_{1} \mid \times 100$ & $2.663 \times 10^{-2}$ & 4.516 & 1.783 & 1.900 & 1.976 & 1.910 & 1.985 \\
\hline & $\mid$ Mean relative bias for $\hat{\beta}_{1} \mid \times 100$ & $2.534 \times 10^{-2}$ & 6.697 & 3.964 & 0.281 & 0.205 & 0.275 & 0.199 \\
\hline & Averaged model-based SE & $4.551 \times 10^{-4}$ & $4.551 \times 10^{-4}$ & $2.459 \times 10^{-2}$ & $4.551 \times 10^{-4}$ & $4.551 \times 10^{-4}$ & $3.828 \times 10^{-4}$ & $3.828 \times 10^{-4}$ \\
\hline & MC SE & $4.808 \times 10^{-4}$ & $2.933 \times 10^{-2}$ & $2.443 \times 10^{-2}$ & $2.133 \times 10^{-2}$ & $2.132 \times 10^{-2}$ & $2.133 \times 10^{-2}$ & $2.132 \times 10^{-2}$ \\
\hline & Relative SE & 0.947 & $1.552 \times 10^{-2}$ & 0.993 & $2.134 \times 10^{-2}$ & $2.135 \times 10^{-2}$ & $1.795 \times 10^{-2}$ & $1.796 \times 10^{-2}$ \\
\hline
\end{tabular}


Table 4. I-G EIV: summary measures for the naive, actual, B-SIMEX, RC, and WRC estimators.

\begin{tabular}{|c|c|c|c|c|c|c|c|c|}
\hline Error size & Measures & Actual & Naive & BSIMEX & $\mathrm{RC}_{\mathrm{E}}$ & $\mathrm{RC}_{\mathrm{A}}$ & $\mathrm{WRC}_{\mathrm{E}}$ & $\mathrm{WRC}_{\mathrm{A}}$ \\
\hline \multirow{6}{*}{$\sigma_{u}=0.023$} & $\mid$ Median relative bias for $\hat{\beta}_{1} \mid \times 100$ & $2.313 \times 10^{-2}$ & 2.978 & 1.497 & 0.343 & 0.354 & 0.132 & 0.136 \\
\hline & $\mid$ Mean relative bias for $\hat{\beta}_{1} \mid \times 100$ & $2.259 \times 10^{-2}$ & 3.375 & 1.888 & $1.929 \times 10^{-3}$ & $1.929 \times 10^{-3}$ & $2.160 \times 10^{-2}$ & $1.716 \times 10^{-2}$ \\
\hline & Averaged model-based SE & $4.588 \times 10^{-4}$ & $5.533 \times 10^{-3}$ & $8.333 \times 10^{-3}$ & $5.549 \times 10^{-3}$ & $5.550 \times 10^{-3}$ & $3.456 \times 10^{-3}$ & $3.456 \times 10^{-3}$ \\
\hline & MC SE & $4.793 \times 10^{-4}$ & $1.192 \times 10^{-2}$ & $8.442 \times 10^{-3}$ & $6.861 \times 10^{-3}$ & $6.087 \times 10^{-3}$ & $3.864 \times 10^{-3}$ & $3.864 \times 10^{-3}$ \\
\hline & Relative SE & 0.957 & 0.464 & 0.987 & 0.912 & 0.912 & 0.894 & 0.894 \\
\hline & Measures & Actual & Naive & BSIMEX & $\mathrm{RC}_{\mathrm{E}}$ & $\mathrm{RC}_{\mathrm{A}}$ & $\mathrm{WRC}_{\mathrm{E}}$ & $\mathrm{WRC}_{\mathrm{A}}$ \\
\hline \multirow{5}{*}{$\sigma_{u}=0.044$} & $\mid$ Median relative bias for $\hat{\beta}_{1} \mid \times 100$ & $2.085 \times 10^{-2}$ & 5.781 & 3.029 & 0.603 & 0.623 & 0.181 & 0.189 \\
\hline & $\mid$ Mean relative bias for $\hat{\beta}_{1} \mid \times 100$ & $2.120 \times 10^{-2}$ & 6.603 & 3.867 & 0.131 & 0.111 & 0.157 & 0.148 \\
\hline & Averaged model-based SE & $4.610 \times 10^{-4}$ & $1.103 \times 10^{-2}$ & $9.223 \times 10^{-2}$ & $1.102 \times 10^{-2}$ & $1.103 \times 10^{-2}$ & $6.724 \times 10^{-3}$ & $6.724 \times 10^{-3}$ \\
\hline & MC SE & $4.806 \times 10^{-4}$ & $2.360 \times 10^{-2}$ & $1.725 \times 10^{-2}$ & $1.222 \times 10^{-2}$ & $1.222 \times 10^{-2}$ & $7.615 \times 10^{-3}$ & $7.614 \times 10^{-3}$ \\
\hline & Relative SE & 0.959 & 0.467 & 5.347 & 0.902 & 0.902 & 0.883 & 0.883 \\
\hline
\end{tabular}




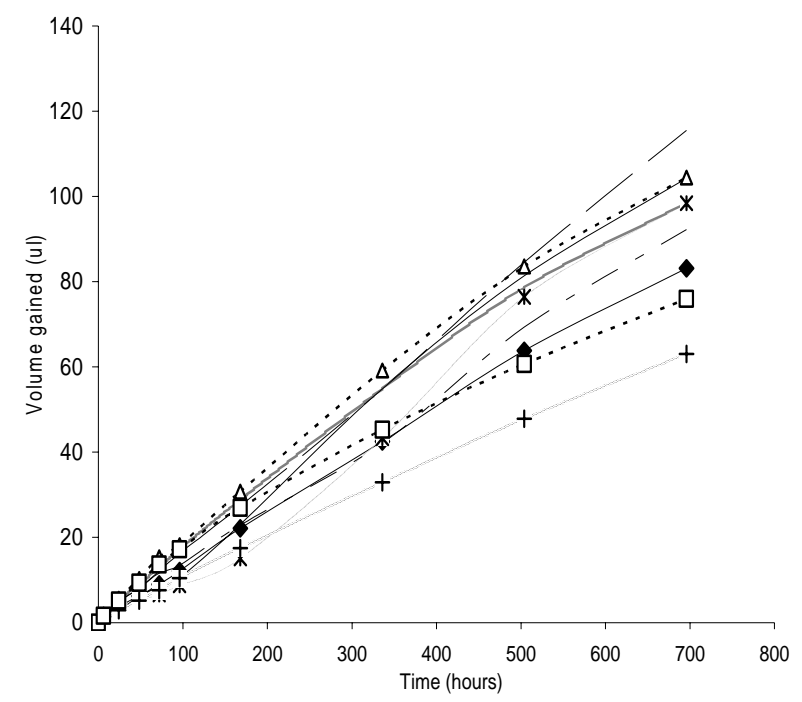

Figure 1. Volume uptake over a period of around 700 hours of 9 compounds stored in a fridge. Each line corresponding to a different compound.

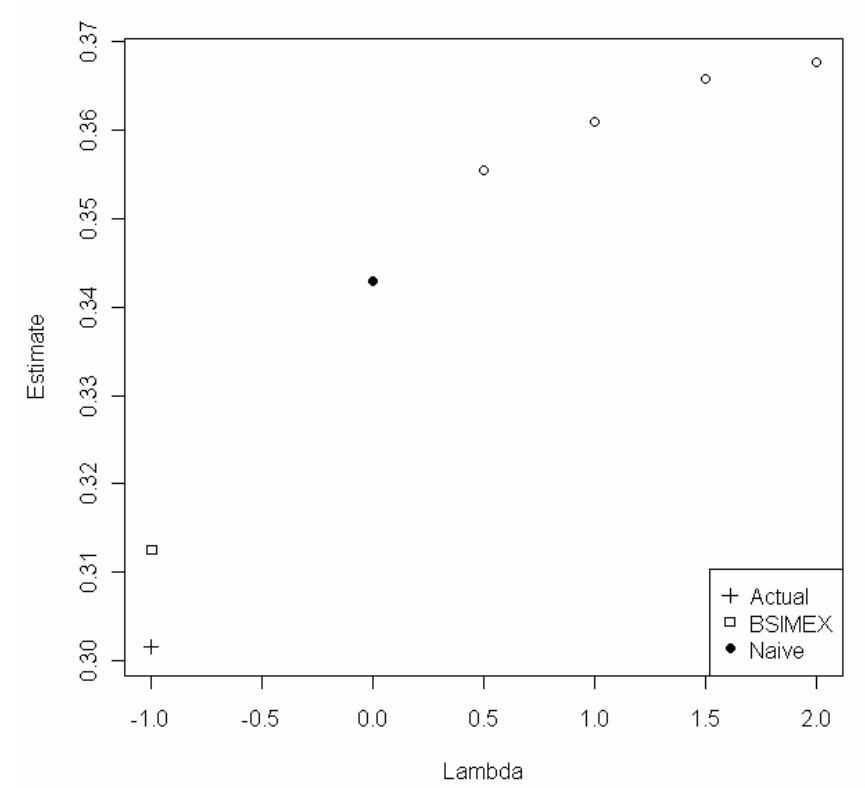

Figure 2. Estimates of $\beta_{1}$ : actual, B-SIMEX, naive and when $\lambda$ is set to $0.5,1,1.5$, and 2 . 